\title{
CHALMERS
}

\section{Bridging the Gap Between Lab Scale and Full Scale Catalysis Experimentation}

\author{
Jonas Sjöblom \\ Applied Mechanics, Chalmers University of Technology, jonas.sjoblom@chalmers.se
}

\section{Introduction}

The knowledge transfer between lab scale and full scale is becoming increasingly important.

Lab scale understanding of complex catalytic phenomena needs to be transferred to full scale with high precision.

Real world issues need to be revisited in more fundamental studies

\section{Objective}

To develop a reactor system (EATS, Emission Aftertreatment System) that creates a bridge between lab scale and full scale research experimentation.

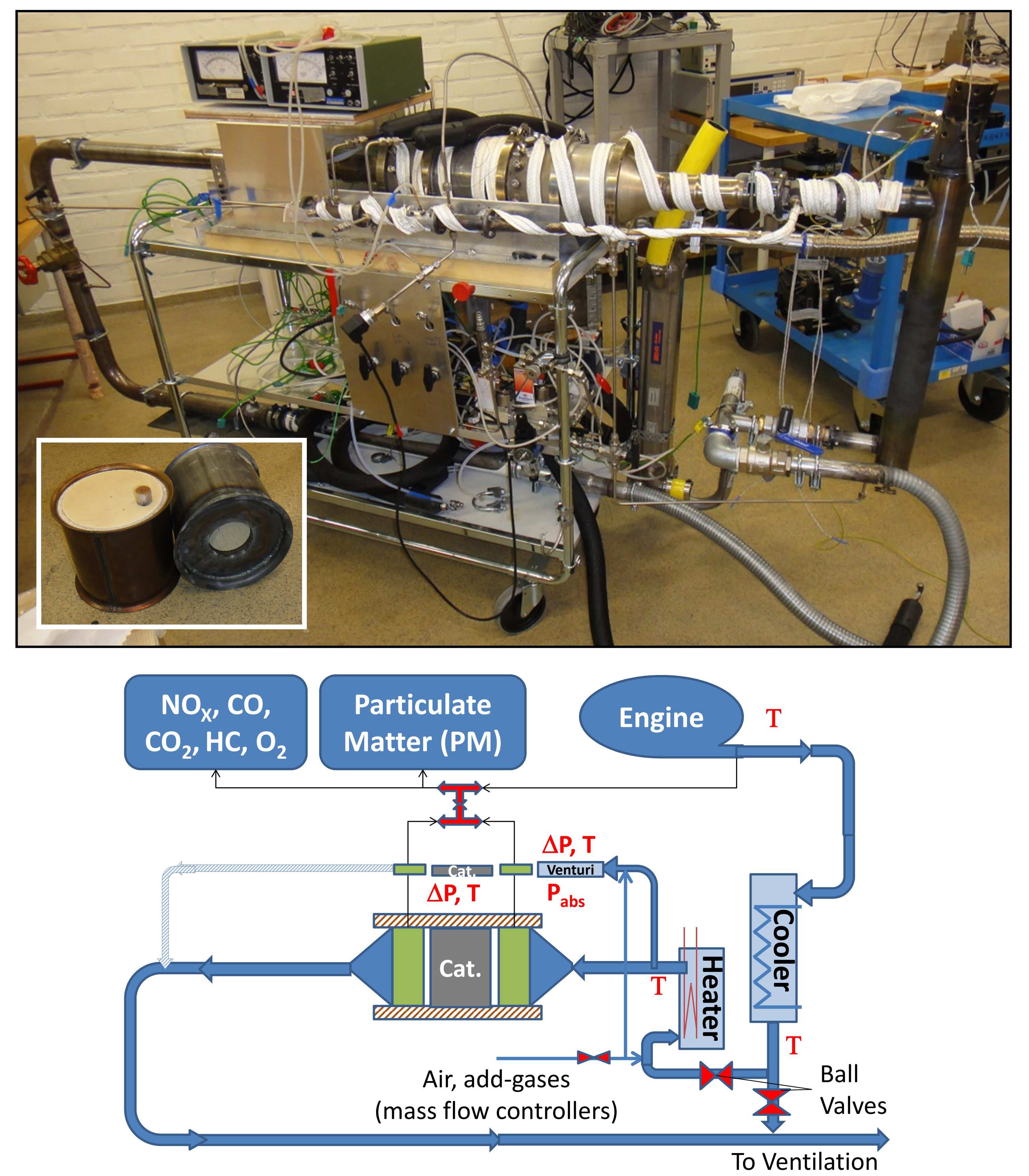

\section{EATS experimental features}

Highly flexible

- Different engine type, size

- Two "legs" (0.2-20 l/min or $10-1000 \mathrm{l} / \mathrm{min}$ )

- Different catalyst sizes

Independent residence time

- Set by flow by-pass (ball valves)

- Measured by pressure drop or venturimeter

Independent concentrations

- Added air or synthetic gases

- Mass flow controllers (Labview)

Independent temperatures

- Cooler \& heater

- "Adiabatic" heating tape

\section{Example 1: Particulate Matter (PM) Capture}

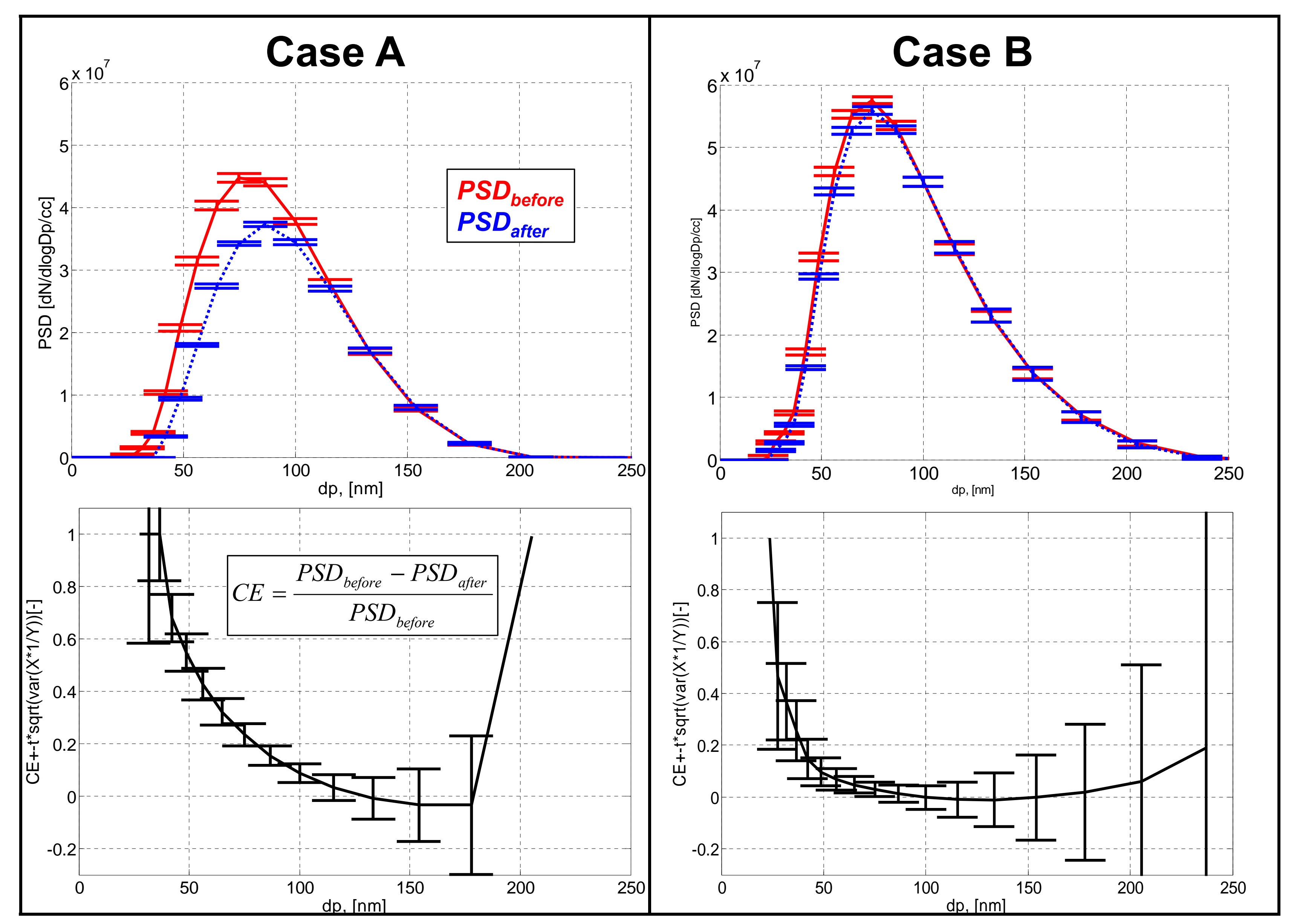

\begin{tabular}{|c|c|c|c|c|c|}
\hline \multicolumn{6}{|c|}{ 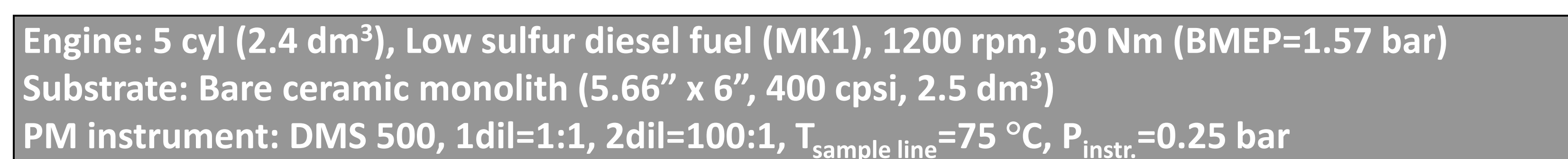 } \\
\hline PM capture conditions & $\begin{array}{l}T_{\text {cat }} \\
{\left[{ }^{\circ} \mathrm{C}\right]}\end{array}$ & $\begin{array}{l}\mathrm{T}_{\min } / \mathrm{T}_{\max } \\
{\left[{ }^{\circ} \mathrm{C}\right]}\end{array}$ & $\begin{array}{l}\text { Flow } \\
\text { [N//min] }\end{array}$ & $\begin{array}{c}\mathrm{Re}_{\text {channel }} \\
{[-]}\end{array}$ & $\begin{array}{l}\mathrm{N} \text { (data point. } \\
\text { before/after }\end{array}$ \\
\hline Case A & 222 & $194 / 258$ & 66 & 2.7 & $185 / 197$ \\
\hline Case B & 157 & $154 / 159$ & 210 & 10.8 & $62 / 110$ \\
\hline
\end{tabular}

\section{Results PM Capture}

- Low flow required to get significant capture

- Capture efficiency (CE) depend on reactor conditions $(T, Q)$

- Uncertainty for CE depends on signal level as well as secondary effects (PM, flow properties, sampling procedures)

\section{Significance}

- Important to measure accurately and representatively

- Secondary effects apparent when comparing with simulations (on-going work)
Example 2: $\mathrm{H}_{2}$-assisted $\mathrm{NH}_{3}$-SCR over $\mathbf{A g}-\mathrm{Al}_{\mathbf{2}} \mathbf{O}_{3}$

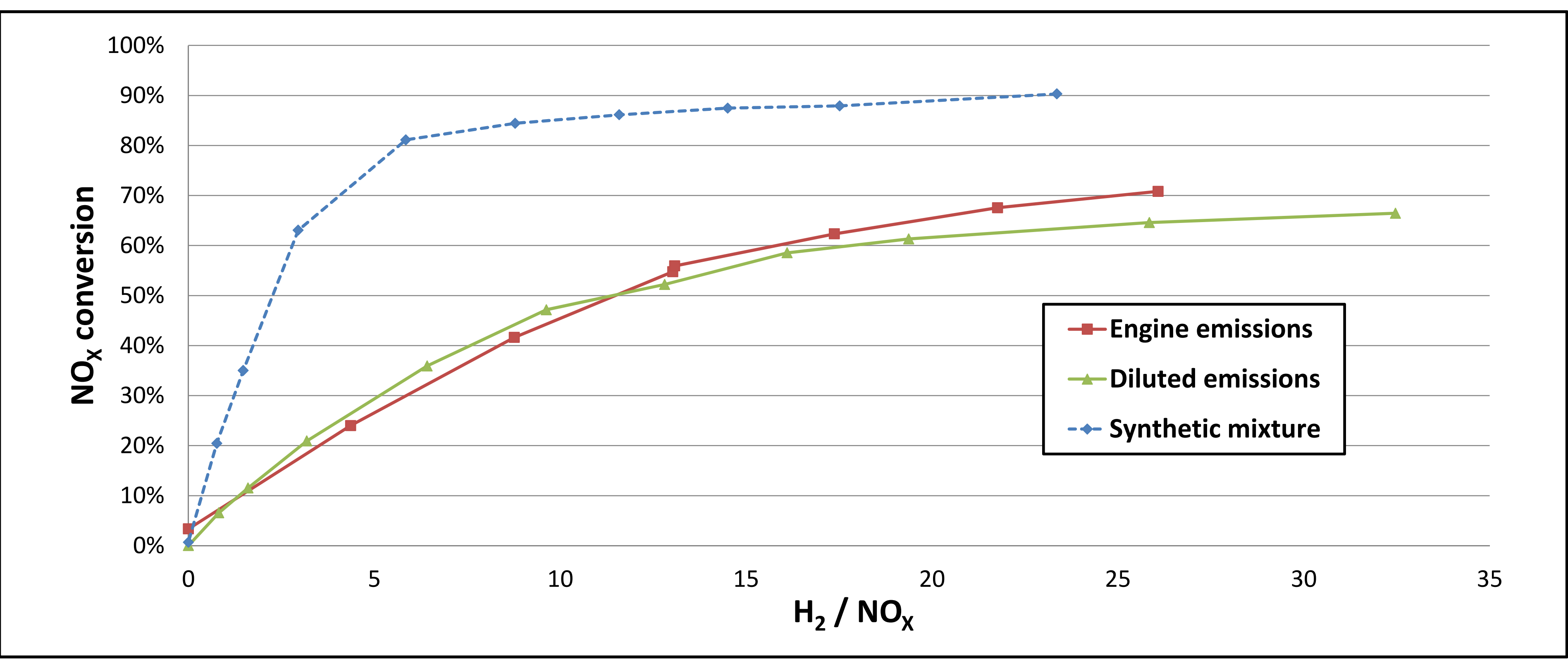

\begin{tabular}{|c|c|c|c|c|c|c|c|c|}
\hline $\begin{array}{|ll|}\text { Engine: } & 1 \mathrm{cyl} \\
\text { Catalyst: } & 2 \times 2 \mathrm{c} \\
\text { Add-gases: } & \mathrm{NH}_{3}(4 \\
& \mathrm{NO}(9\end{array}$ & $\begin{array}{l}\left.\mathrm{m}^{3}\right) \text {, Dies } \\
\left(8 \mathrm{~cm}^{3}\right), A \\
\left.\mathrm{~N}_{2}\right), \mathrm{H}_{2}(4 \mathrm{(} \\
\left.\mathrm{m} \text { in } \mathrm{N}_{2}\right) 1\end{array}$ & $\begin{array}{l}\text { fuel (MK } \\
\left(\mathrm{Al}_{2} \mathrm{O}_{3}: 2:\right. \\
\% \text { in } \mathrm{He}), \\
r \text { syntheti }\end{array}$ & $\begin{array}{l}1,1200 \mathrm{rpm}, \\
\mathrm{N} / \mathrm{w} \text { (freeze } \\
\text { mpressed d } \\
\text { mixture }\end{array}$ & $\begin{array}{l}4.7 \mathrm{Nm} \\
\text { dried } \\
\text { va air }\end{array}$ & $\begin{array}{l}\text { MEP=3. } \\
\text { el prepa } \\
\text { luted en }\end{array}$ & $\begin{array}{l}\text { bar) } \\
\text { tion) } \\
\text { sions }\end{array}$ & & \\
\hline Inlet conditions & $\mathrm{T}_{\text {cat }}\left[{ }^{\circ} \mathrm{C}\right]$ & $\begin{array}{c}\text { Flow } \\
\text { [NI/min] }\end{array}$ & $\mathrm{NO}_{\mathrm{x}}[\mathrm{ppm}]$ & $\begin{array}{c}\mathrm{NH}_{3} \\
{[\mathrm{ppom}}\end{array}$ & $\mathrm{H}_{2} \mathrm{O}[\%]$ & $\begin{array}{c}\mathrm{CO} \\
{[\mathrm{ppm}]}\end{array}$ & $\mathrm{CO}_{2}[\%]$ & $\begin{array}{c}\text { "Diesel" } \\
\left(C_{1}\right)[p p m]\end{array}$ \\
\hline Engine emissions & 255 & 1.6 & 548 & 451 & 3.5 & 150 & 4.2 & 56 \\
\hline Diluted emissions & 256 & 1.6 & 376 & 264 & 3.0 & 102 & 2.8 & 52 \\
\hline ynthetic mixture & 252 & 0.6 & 293 & 234 & $v$ & 0 & 0 & 0 \\
\hline
\end{tabular}

\section{Results $\mathbf{A g}-\mathrm{Al}_{\mathbf{2}} \mathbf{O}_{\mathbf{3}} \mathbf{S C R}$}

- Same $\mathrm{H}_{2}$ effect upon dilution if conversion evaluated as $\mathrm{H}_{2}$ to $\mathrm{NO}_{\mathrm{x}}$ ratio

- Synthetic mixture had different residence time, which affects both $\mathrm{NO}_{\mathrm{X}}$ conversion and un-selective $\mathrm{H}_{2}$ reactions

\section{Significance}

- Same catalyst evaluated at both lab scale and engine conditions

- Important comparison with lab scale results (on-going work)

\section{Conclusions}

The EATS enables experimentation that is not possible using traditional methods. 